Mens

revue d'histoire intellectuelle de l'Amérique française

\title{
Les dix ans de Mens
}

\section{Guy Laperrière}

Volume 11, numéro 1, automne 2010

URI : https://id.erudit.org/iderudit/1023336ar

DOI : https://doi.org/10.7202/1023336ar

Aller au sommaire du numéro

Éditeur(s)

Centre de recherche en civilisation canadienne-française

ISSN

1492-8647 (imprimé)

1927-9299 (numérique)

Découvrir la revue

Citer cet article

Laperrière, G. (2010). Les dix ans de Mens. Mens, 11(1), 9-35.

https://doi.org/10.7202/1023336ar

Ce document est protégé par la loi sur le droit d'auteur. L'utilisation des services d'Érudit (y compris la reproduction) est assujettie à sa politique d'utilisation que vous pouvez consulter en ligne.

https://apropos.erudit.org/fr/usagers/politique-dutilisation/
Cet article est diffusé et préservé par Érudit.

Érudit est un consortium interuniversitaire sans but lucratif composé de l’Université de Montréal, l'Université Laval et l'Université du Québec à Montréal. Il a pour mission la promotion et la valorisation de la recherche. https://www.erudit.org/fr/ 


\title{
Perspective
}

\section{Les dix ans de Mens}

\author{
Guy Laperrière \\ Département d'histoire \\ Université de Sherbrooke
}

L'équipe de Mens m'a demandé de rédiger un bilan des dix ans de la revue et du champ de l'histoire intellectuelle. Je me suis aussitôt récusé : ce n'est pas là mon domaine, et d'autres sont bien plus qualifiés que moi pour mener à bien cette entreprise. J'eus beau dire, on revint à la charge. Et j'ai accepté, parce que je suis la revue avec intérêt depuis le début, que l'équipe est dynamique et que le défi mérite d'être relevé. Mais plutôt qu'à de savantes réflexions sur la substantifique moelle de l'histoire intellectuelle, je convie le lecteur à un parcours rétrospectif des dix premières années de la revue, de 2000 à 2010. Je me permettrai de prendre un ton personnel : on aura là les impressions d'un lecteur attentionné.

Le premier souvenir que je garde de l'apparition de la revue Mens est double. Me revient en mémoire l'image de deux ou trois étudiants zélés, assis à une misérable table du congrès de l'Institut d'histoire de l'Amérique française, en octobre 2000, et qui vendaient le premier numéro d'une revue, bien chétive dans son petit format et ses quatrevingts pages à la typographie trop serrée. Le numéro se vendait $10 \$$; l'abonnement, $15 \$$. Et longtemps après le départ des exposants de livres, le samedi midi, nos dévoués zélateurs continuèrent à discuter avec qui voulait bien les entendre. On voyait que c'étaient des étudiants d'histoire passionnés. Quel avenir allait connaître cette petite 
revue d'étudiants ? Rares sont ceux qui, alors, auraient misé sur sa pérennité.

Mon deuxième souvenir tient à mes hautes fonctions : j'étais alors président de l'Institut d'histoire de l'Amérique française, dont je devais manifestement défendre les intérêts, en premier lieu ceux de la docte Revue d'histoire de l'Amérique française (RHAF). Or, n'y avait-il pas injure, sinon attentat, à donner à cette nouvelle Revue d'histoire intellectuelle de l'Amérique française le sigle de RHLAF, lequel se confond à vue d'œil avec celui de $R H A F$ ? Je me fendis alors d'une belle lettre et reçus réponse, dans le style de la fable Le loup et l'agneau, que d'aucune façon on ne voulait troubler le cours de cette noble revue et qu'on s'empresserait, à la première occasion, de modifier ce sigle qui pouvait être source de confusion. Comme de fait, le sigle de l'adresse internet se transforma en "revuemens», et la direction de la $R H A F$ put retrouver le sommeil.

Je lus ce premier numéro pour m’apercevoir que c'était l'œuvre d'étudiants de maîtrise de Pierre Trépanier, de l'Université de Montréal. Lionel Groulx était à l'honneur, comme il se doit, le premier article reprenant le mémoire de Damien-Claude Bélanger sur la francoaméricanie. Il y avait aussi un article d'un autre membre de l'équipe, Dominique Foisy-Geoffroy, sur Esdras Minville : c'étaient aussi des éléments de son mémoire de maîtrise. Un troisième article traitait de Pierre Elliott Trudeau, toujours par une candidate à la maîtrise. Le ton était donné. Le mois suivant, Louis Cornellier publiait dans sa chronique du Devoir ${ }^{1}$ un compte rendu favorable de cette dernièrenée de nos revues d'histoire.

\section{Le contexte de fin de siècle}

Dans quel contexte apparaissait cette nouvelle revue ? C'était la période de l'après-référendum (de 1995), et la scène intellectuelle en histoire était en effervescence. L'événement, c'était le livre de Ronald

\footnotetext{
1 Louis Cornellier, "De jeunes historiens des idées ", Le Devoir, 18-19 novembre 2000, p. D18.
} 
Rudin, Making History in Twentieth-Century Quebec ${ }^{2}$ (1997), ouvrage traduit dès l'année suivante avec un titre raccourci : Faire de l'histoire au Québec. La traduction du titre en français laissait échapper la thèse de l'auteur, le mot making ayant plutôt ici le sens de "fabriquer" l'histoire. On se souvient, en gros, que cet ouvrage venait réhabiliter Lionel Groulx et critiquer la génération de l'école qu'on appela d'abord révisionniste, puis, d'un terme plus approprié, moderniste, qui avait laissé dans l'ombre plusieurs traits importants de l'histoire du Québec. Et les répliques de fuser de toutes parts, notamment dans le Bulletin d'histoire politique, dont l'une des vocations était de provoquer et d'animer les débats.

\section{Le monde des revues d'histoire}

On aurait pu croire que la revue Mens venait prendre la succession des Cahiers d'histoire du Québec au XXe siècle, revue semestrielle, elle aussi, née en 1994 et qui n'en finissait plus d'agoniser lorsqu'elle publia son dixième et dernier numéro en janvier 2000, sur André Laurendeau - le numéro précédent avait paru en 1998. Lancée par le père Benoît Lacroix, qui avait amassé les fonds de départ et qui portait le titre de directeur, cette revue, de bonne tenue, était, en fait, l'œuvre de son rédacteur en chef, Stéphane Stapinsky, lui-même apparenté à Groulx et œuvrant au Centre de recherche Lionel-Groulx, lequel se débattait, comme toujours, dans les problèmes de manque de fonds et publiait la revue. La logistique était assez lourde, et on peut penser que cette revue a disparu par manque d'argent. C'est d'ailleurs ce qu'affirment les deux principaux responsables dans la présentation du dernier numéro, soulignant, par ailleurs, l'apparition, en 1998, de deux nouvelles revues, Argument et Globe.

Il suffit cependant de jeter un coup d'œil sur Les Cabiers et sur Mens pour s'apercevoir que les personnes actives dans l'une et l'autre

2 Ronald Rudin, Making History in Twentieth-Century Quebec, Toronto, University of Toronto Press, 1997 ; Faire de l'histoire au Québec, trad. Pierre R. Desrosiers, Sillery, Septentrion, 1998. 
revue ne sont pas du tout les mêmes. Donc, succession dans le temps, mais aucune filiation. Par contre, Les Cahiers ont pu contribuer à effectuer une trouée dans le monde des revues dans laquelle Mens allait s'avancer. En 2003, Stéphane Kelly les présente ainsi : "Cette revue a joué un rôle important durant cette décennie en contestant l'hégémonie des revues académiques sur la réflexion historique, notamment en revalorisant l'histoire culturelle, l'histoire intellectuelle et l'histoire religieuse ${ }^{3}$."

Peut-on continuer à brosser le tableau des principales revues d'histoire au Québec en cette fin de siècle ? Au sommet du firmament trône la reine de nos revues d'histoire, la Revue d'histoire de l'Amérique française, dirigée de main de maître de 1996 à 2000 par Fernande Roy, qui la quitta en lui léguant une nouvelle maquette et une nouvelle mise en page. En 1997, pour son cinquantenaire, la revue a publié, sous la responsabilité de Jacques Rouillard, un numéro spécial sur "Les pratiques de l'histoire de l'Amérique française depuis 50 ans ", duquel je retiens, à côté d'un stimulant article de Gérard Bouchard sur "L'histoire sociale au Québec ", le bilan d'Yvan Lamonde sur "L'histoire culturelle comme domaine historiographique au Québec", un solide article qui fait bien le tour de la question et permet de situer l'histoire intellectuelle. J'y reviens dans un instant.

Parmi les autres revues d'importance, signalons la Canadian Historical Review, qui a pris, avec une nouvelle couverture toute rouge, un nouvel élan lors de sa $75^{\mathrm{e}}$ année, en 1995 . Un membre du Québec siège au comité de rédaction : ce fut Jean-Marie Fecteau de 1995 à 1998, puis Peter Gossage, qui fut aussi codirecteur de 2000 à 2003. L'année 2003, surtout, vit paraître trois ou quatre articles sur le Québec. Parmi les autres revues d'histoire générale, on peut citer Histoire sociale, la Revue de la Société historique du Canada et Les Cahiers des Dix. Pierre Trépanier et Yvan Lamonde sont de la Société des Dix depuis 1988 et 2000 respectivement.

3 Stéphane Kelly, Les idées mènent le Québec : essais sur une sensibilité historique, Québec, Les Presses de l'Université Laval, 2003, p. 1. 
Mens prend évidemment rang parmi les revues d'histoire thématiques, qui se sont multipliées depuis 1970. Sans vouloir être exhaustif, citons Recherches amérindiennes au Québec (1971), Revue d'histoire urbaine (1972) - plusieurs d'entre elles ont un titre bilingue, nous ne citons ici que le français -, Labour / Le travail (1976) - je fais ici exception, car cette revue ne publie en pratique qu'en anglais -, Bulletin canadien d'histoire de la médecine (1984), Revue d'histoire de l'éducation (1989), une revue authentiquement bilingue, celle-là, Études d'histoire religieuse, nouveau titre (1990) d'une revue plus ancienne, qui fait peau neuve à ce moment-là. Or, il est frappant de constater que l'apparition de chacune de ces revues marque l'entrée en force de la sous-discipline correspondante dans le champ historique. Avec Mens, la plus récente de ces revues - je n'en connais pas d'autre depuis - c'est l'histoire intellectuelle qui prend sa place dans l'histoire du Québec.

Il faut faire une place à part au Bulletin d'histoire politique (1992), qui a entrepris de donner un autre ton à une revue savante d'histoire, un ton moins savant, justement, plus engagé, avec des articles courts, sur des thèmes faisant l'objet de débats dans l'actualité, des numéros thématiques, des chroniques régulières, un format plus convivial. La revue veut redonner place à des pans de l'histoire plutôt délaissés, notamment l'histoire politique, l'histoire militaire, l'histoire ouvrière. Elle est l'œuvre d'une équipe de l'Université du Québec à Montréal, traditionnellement plus engagée, moins haut de gamme que les nobles universités McGill ou de Montréal, perchées sur la montagne. Ce ne sera certes pas le modèle de Mens, mais le fait que le Bulletin guerroie dans le monde des idées et lance des débats rend plus pertinente une revue d'histoire intellectuelle comme Mens.

On pourrait aussi citer d'autres initiatives qui aident à mieux comprendre le contexte des revues d'histoire à cette période précise. Cap-aux-Diamants, un magazine trimestriel de vulgarisation de l'histoire, est apparu en 1985 à Québec, œuvre de diplômés d'histoire de l'Université Laval. La revue Globe, de l'Université McGill, renaît en 1998, se décrivant comme une "revue internationale d'études 
québécoises " rattachée au programme d'études sur le Québec de l'Université McGill. Enfin, les étudiants du Département d'histoire de l'Université de Montréal ont lancé en 1981 des Cahiers d'histoire, qui ont beaucoup évolué avec le temps. Une recherche sommaire dans les numéros entourant l'apparition de Mens m'a permis d'y retrouver un article de Michel Ducharme en 1998 et un autre de Dominique Foisy-Geoffroy en 2000, tous deux portant sur leur sujet de maittrise (Lord Durham, Esdras Minville). On le voit : les initiateurs de Mens ne parlent pas sans raison, dans le premier paragraphe de la présentation du premier numéro, du " nombre important de revues d'histoire déjà en circulation ". Cela ne les a pas empêchés de très bien camper leur niche dans le paysage.

\section{Le paysage de l'histoire intellectuelle au Québec}

L'histoire intellectuelle connaît alors une certaine vogue au Québec. Depuis la fin des années 1980 et le début des années 1990, l'histoire culturelle a pris le relais de l'histoire sociale comme principal vecteur de la discipline historique. De même qu'entre 1965 et 1985 , tout était histoire sociale, à partir de 1990, tout devient histoire culturelle : l'histoire de l'éducation, l'histoire religieuse, l'histoire du livre et de l'imprimé, l'histoire littéraire, l'histoire de l'art et, à combien plus forte raison, l'histoire intellectuelle et l'histoire des idées.

C'est le moment de revenir à la synthèse très complète qu'en a faite son principal initiateur au Québec, Yvan Lamonde, dans le numéro de la $R H A F$ de 1997 que nous mentionnions à l'instant. Il y distingue trois phases de la recherche. La première, dans les années 1960, est celle de l'étude des influences culturelles de la France sur le Québec : on pense à l'ultramontanisme ou au libéralisme. Très voisins, viennent ensuite les travaux sur ces mêmes courants de pensée au Québec même, au XIx ${ }^{\mathrm{e}}$ siècle surtout : ce fut la grande mode de l'histoire des idéologies, au début des années 1970. Enfin, on étudie différentes formes ou différents canaux par lesquels s'exprime la culture : le collège classique (Claude Galarneau), l'imprimé, les 
bibliothèques, la librairie, les associations (Yvan Lamonde), la presse (Jean de Bonville). Parmi les questions débattues, Lamonde ne manque pas de signaler son refrain favori : la permanence d'une tradition libérale anticléricale au Québec, dont deux de ses ouvrages parus presque simultanément donnent tout à fait le ton : sa grande biographie de Louis-Antoine Dessaulles (Fides, 1994), son héros par excellence - dont le sous-titre dit bien l'ambition : un seigneur libéral et anticlérical -, et le collectif qu'il a dirigé sur les Combats libéraux au tournant $d u X X$ siècle (Fides, 1995), où défilent tour à tour les vaillants résistants à l'emprise de la censure cléricale, de Wilfrid Laurier à Laurent-Olivier David, de la loge L'Émancipation au Pays de Godfroy Langlois ou à L'Autorité de Gaston Maillet. Il appelle de ses vœux des travaux plus poussés sur l'histoire intellectuelle de l'entredeux-guerres, où les débats enflammés - on pense à l'antisémitisme - ont fait rage sans que toujours la recherche sereine soit au rendez-vous.

Citons, en terminant, une caractérisation un peu restrictive que fait Lamonde de l'histoire intellectuelle. Après avoir affirmé péremptoirement que " l'histoire intellectuelle ne pouvait être qu'une province d'une histoire culturelle plus générale parce que plus englobante socialement ", il poursuit : "Alors que l'histoire culturelle englobe tous les groupes sociaux et toutes leurs formes d'expression ou de langages - autant l'écrit, l'éloquence, l'oralité de la culture populaire, la gestuelle, la culture matérielle -, l'histoire intellectuelle privilégie l'écrit et une certaine expression verbale ${ }^{4}$."

La même année, en mars 1997, se tenait à l'Université du Québec à Trois-Rivières, organisé par Manon Brunet et Pierre Lanthier, un colloque dont les actes allaient être publiés en 2000 sous le même titre, L'inscription sociale de l'intellectuel (Les Presses de l'Université Laval, L'Harmattan, 2000). Il y avait là une vingtaine de chercheurs de pays et d'horizons fort divers. Je relève quelques communications,

\footnotetext{
4 Yvan Lamonde, "L'histoire culturelle comme domaine historiographique au Québec ", Revue d'histoire de l'Amérique française, vol. 51, n 2 (automne 1997), p. 288.
} 
qui donnent bien l'atmosphère qui vit naître Mens : Michel Trebitsch (CNRS, Paris), sur les intellectuels et l'idée européenne dans l'entredeux-guerres ; Yvan Lamonde, sur les intellectuels au Québec entre 1900 et 1930 ; Catherine Pomeyrols, sur les milieux de formation des intellectuels de l'entre-deux-guerres ; Fernande Roy, sur les journalistes canadiens-français du $\mathrm{xx}^{\mathrm{e}}$ siècle, qu'elle souhaitait d'emblée ranger parmi les intellectuels. La revue Mens arrivait donc au bon moment.

\section{Les premiers numéros : l'investissement d'un champ}

De 2000 à 2002, dans ses cinq premiers numéros, la revue cherche sa voie. Non que l'équipe ne sache où elle va, bien au contraire. Mais les auteurs, les lecteurs, doivent s'habituer à cette nouvelle revue. L'équipe elle-même se solidifie, autour d'un noyau central. Les types d'articles, plus variés au début, avec des perspectives plus libres, des prises de position sur des sujets d'actualité, se resserrent, et la revue trouve progressivement son créneau. On peut dire qu'à partir de 2003, elle a vraiment pris son erre d'aller.

Des cinq membres du premier comité de direction, trois en constitueront le noyau et ont tenu le fort pendant les dix premières années : Yves Bégin, Damien-Claude Bélanger et Dominique FoisyGeoffroy. À partir de 2002, trois autres membres se joignent à l'équipe, qui resteront en poste jusqu'à 2009 : ce sera là le comité de rédaction qui donnera à la revue son visage de ces années. Il s'agit de Xavier Gélinas, disciple le plus achevé de Pierre Trépanier, avec sa thèse sur La droite intellectuelle québécoise et la Révolution tranquille, 1956-1966, dirigée par Ramsay Cook à l'Université York, soutenue en 2001 et publiée aux Presses de l'Université Laval en 2007 ; Michel Bock, qui venait de déposer sa thèse sur Les minorités françaises dans la pensée de Lionel Groulx, qui sera publiée chez Hurtubise HMH en 2004 ; et Michel Ducharme, alors doctorant à l'Université McGill, dont la thèse vient de paraître sous le titre Le concept de liberté au Canada à l'époque des révolutions atlantiques, 1776-1838 (McGill-Queen's 
University Press, 2010). Ces six membres du comité de rédaction montrent bien l'une des principales caractéristiques de la revue, sinon de l'histoire intellectuelle elle-même : elle est entièrement masculine.

Partis de l'Université de Montréal avec leur maîtrise, les initiateurs de Mens se dirigent vers leur doctorat surtout dans deux directions : l'Université McGill (Bélanger, Ducharme) et l'Université Laval (Bégin, Foisy-Geoffroy). À McGill, autour de l'Institut d'études canadiennes, Bélanger et Ducharme organisent, en mars 2003, avec Sophie Coupal, un symposium bilingue : "Nouvelles orientations en histoire intellectuelle du Canada ». Les actes de ce symposium seront publiés l'année suivante aux Presses de l'Université Laval, sous le titre Les idées en mouvement : perspectives en histoire intellectuelle et culturelle du Canada, dans une collection dirigée par Yvan Lamonde. Les jeunes organisateurs ont fait un grand effort pour faire dialoguer les spécialistes de ce champ, tant au Québec qu'au Canada anglais. C'est ainsi qu'à côté des Jocelyn Létourneau, Pierre Trépanier ou Yvan Lamonde, on retrouvait les Ramsay Cook, A. B. McKillop ou Cecilia Morgan. Ce colloque était une première manifestation de cette année 2003, qui allait constituer un sommet dans l'histoire intellectuelle.

Quant à l'Université Laval, son Département d'histoire, et en particulier la Chaire de recherche du Canada en histoire et économie politique du Québec contemporain de Jocelyn Létourneau, de même que ses presses, allaient jouer un rôle déterminant dans l'évolution de la revue. À partir de 2002, cette dernière est publiée avec l'appui technique des Presses de l'Université Laval et des subventions et divers services du Département et de la Chaire. Elle a désormais trouvé son créneau : les articles sont évalués par des lecteurs externes, et son activité veut se déployer "d'abord et avant tout dans le champ de l'histoire érudite " "à partir le plus souvent de recherches universitaires. Ce qui n'exclut pas d'intéresser un public cultivé, « soucieux d'enrichir

5 Dominique Foisy-Geoffroy (Pour l'équipe de Mens), "Présentation ", Mens : revue d'histoire intellectuelle de l'Amérique française, vol. II, $\mathrm{n}^{\circ} 2$ (printemps 2002), p. 149. 
[sa] compréhension du monde ${ }^{6} "$. Comme toujours dans leurs présentations, les responsables sont enthousiastes et de bonne humeur : "Bref, les choses vont bon train $[\ldots]^{7}$ "

\section{Un état des lieux français : L'bistoire des intellectuels aujourd'bui}

En mars 2003 était publié aux Presses universitaires de France un collectif sous la direction de Michel Leymarie et Jean-François Sirinelli, ouvrage issu d'un colloque tenu les 16 et 17 octobre 2001 visant à faire le point sur L'histoire des intellectuels aujourd'hui. Les séances étaient présidées par cinq " autorités "; outre les deux déjà nommés, s'ajoutaient Christophe Charle, Pascal Ory et Jean-Yves Mollier, tous des hommes encore - notons-le au passage -, et tous (sauf Leymarie) d'une même génération, nés entre 1948 et 1951, de même d'ailleurs que François Dosse, l'un des intervenants principaux. Outre l'objectif principal de produire un état des lieux, l'un des buts du colloque était de passer la main à la génération suivante, appelée à faire les présentations.

Après avoir fait remarquer que l'histoire intellectuelle se situe "à la croisée des histoires politique et socioculturelle ", le collectif s'ouvre sur une tournée de l'historiographie dans plusieurs pays, sur le thème "Comment fait-on l'histoire intellectuelle à l'étranger ?". Sont passés en revue l'Italie, l'Allemagne, l'Angleterre, l'Espagne, l'Argentine et, heureuse surprise, le Québec, avec une présentation substantielle de Catherine Pomeyrols, "Comment fait-on l'histoire des intellectuels au Québec ? ». Elle y aborde le paradigme de la modernité, fait une revue de l'histoire culturelle (la littérature, la presse, l'article de Lamonde de 1997) ; elle passe ensuite à l'interprétation de la Révolution tranquille, dont l'article et l'ouvrage de Rudin, pour terminer par les liens avec la France. Plus loin sont présentés des travaux faisant état des recherches en Belgique et en

${ }^{6}$ Ibid., p. 150.

7 Ibid. 
Suisse, et la dernière section fournit l'occasion de "regards croisés" entre ces différents pays, auxquels on ajoutera les États-Unis.

La deuxième partie, pour sa part, traite de l'apport des disciplines voisines : édition (Mollier), histoire littéraire, sociologie, médias. Retenons en particulier un bon article de François Dosse, qui fait le point sur l'histoire des idées - on peut penser ici à la philosophie -, toujours un peu suspecte en France. Cet article s'intitule d'ailleurs "De l'histoire des idées à l'histoire intellectuelle » et appelle à la barre des auteurs comme (parmi d'autres) Michel Foucault ou Michel de Certeau, Jean Touchard, Paul Ricœur, Robert Darnton ou Roger Chartier, Jean-François Sirinelli ou Michel Trebitsch, sans oublier François Dosse lui-même. Sa conclusion ? «Un vaste chantier d'investigation s'ouvre à de nouvelles convergences entre l'histoire de la pensée et l'histoire tout court ${ }^{8}$."

\section{Un sommet pour l'histoire intellectuelle : l'année 2003}

Le symposium de McGill était un exercice bien sage au regard des éruptions volcaniques qui allaient marquer l'histoire intellectuelle au Québec durant l'année 2003. L'année avait débuté avec la publication d'un collectif dirigé par Stéphane Kelly, fruit d'un autre colloque organisé à l'Université McGill par le programme d'études sur le Québec en mars 2000, sur " la mutation de la sensibilité historique", publié sous le titre Les idées mènent le Québec : essais sur une sensibilité historique (Les Presses de l'Université Laval, 2003). Sous la plume de Thierry Nootens', le Bulletin d'histoire politique le descendit en flammes, le présentant comme une charge passéiste. Dans Mens (automne 2003 également), Joseph Yvon Thériault y vit « un recueil d'histoire

8 François Dosse, "De l'histoire des idées à l'histoire intellectuelle ", dans Michel Leymarie et Jean-François Sirinelli (dir.), L'histoire des intellectuels aujourd'hui, Paris, Presses universitaires de France, 2003, p. 181.

9 Thierry Nootens, "Les idées mènent le Québec ?" Sur une nouvelle "sensibilité historique" et ses apories ", Bulletin d'histoire politique, vol. 12, n 1 (automne 2003), p. 161-169, avec la collaboration de Jean-Marie Fecteau. 
des idées qui arrive à point ${ }^{10}$ ". La nouvelle sensibilité, expliquait-il, cherche à faire " une lecture du Québec plus sympathique au passé [...], pour contrer la désinvolture des modernistes contre la tradition ${ }^{11}$ ». Éric Bédard, Xavier Gélinas, E.-Martin Meunier et Jean-Philippe Warren comptaient parmi les auteurs du recueil qui illustraient le mieux ces " dialogues avec la tradition".

Au même moment, issu lui aussi d'un séminaire tenu en 2001, paraît, en 2003, un collectif dirigé par Marie Christine Weidmann Koop, Le Québec aujourd'hui : identité, société et culture (Les Presses de l'Université Laval), qui s'ouvre sur un article d'Éric Bédard, «Modernité et histoire des idées au Québec : une perspective historiographique". L'auteur y fait état d' " une nouvelle sensibilité ", dont l'essai d'E.-Martin Meunier et Jean-Philippe Warren Sortir de la "Grande Noirceur " : l'horizon "personnaliste " de la Révolution tranquille (Septentrion, 2002) apparaît comme la meilleure illustration.

Par un alignement des astres dont il faudrait chercher l'origine, toute une série d'études sur Lionel Groulx paraissent en 2003. Mens y fera écho dès son numéro du printemps 2004, alors que Pierre Trépanier publie une note critique de 35 pages sur " la luxuriance des études groulxiennes ". Il y présente, notamment, l'ouvrage de Marie-Pier Luneau, Lionel Groulx : le mythe du berger (Leméac, 2003), une fine analyse des stratégies d'auteur de Groulx à partir de sa correspondance ; celui de Frédéric Boily, La pensée nationaliste de Lionel Groulx (Septentrion, 2003), définie comme un "nationalisme organiciste ", livre que Trépanier trouve " intelligent et serein ${ }^{12}$ ", par contraste, de toute évidence, avec le livre qu'il analyse ensuite, Les deux chanoines (Boréal, 2003) de Gérard Bouchard, qu'il critique

${ }^{10}$ Joseph Yvon Thériault, "Les idées mènent le Québec, sous la direction de Stéphane Kelly ", Mens : revue d'histoire intellectuelle de l'Amérique française, vol. IV, $\mathrm{n}^{\circ} 1$ (automne 2003), p. 102.

11 Ibid., p. 99.

12 Pierre Trépanier, "Le renard ayant la queue coupée ou La luxuriance des études groulxiennes (1999-2003)", Mens : revue d'histoire intellectuelle de l'Amérique française, vol. IV, $\mathrm{n}^{\circ} 2$ (printemps 2004), p. 286. 
vertement, non sans y avoir trouvé cependant « du bon or, pur et massif ${ }^{13}$ ". Il a été ébloui par la thèse de Norman F. Cornett, sur le rôle de la religion dans la pensée nationaliste de Groulx (McGill, 2002), thèse qui n'a cependant pas été publiée. Ce même numéro contient une appréciation de ce même ouvrage de Gérard Bouchard par Ramsay Cook, qui appelait de ses vœux une biographie documentée de Groulx, qui lui apparaissait comme " une nécessité fondamentale ${ }^{14}$ " et qu'on espère toujours.

Les deux chanoines n'avait pas fini de faire couler de l'encre. Fernande Roy, dans Études d'histoire religieuse (2005) n'en laisse pas pierre sur pierre. C'est peut-être Michel Bock, dans Histoire sociale (novembre 2004), qui tire la conclusion la plus pondérée : "Il faut don' se demander si certaines des contradictions que trouve l'auteur dans la pensée de Groulx n'auraient pas été tempérées s'il les avait replacées plus clairement à l'intérieur du contexte intellectuel dans lequel a évolué le chanoine ${ }^{15}$."

Saisissant la balle au bond, le Centre de recherche Lionel-Groulx et son directeur général Robert Boily décidèrent de tenir, le 8 novembre 2003, un colloque, dont les actes furent publiés en 2005, sur $U n$ héritage controversé : nouvelles lectures de Lionel Groulx (VLB éditeur). Les auteurs précités vinrent y présenter leur livre, et la journée se termina par une analyse des Deux chanoines par Pierre Trépanier, d'un ton que je trouvai, pour ma part, peu amène. Le livre reproduit les échanges entre Bouchard et Trépanier, que pimente une intervention de Fernande Roy qui tente de ramener les échanges sur le terrain des idées. On trouve, à la fin de l'ouvrage, la liste des participants à ce colloque : inutile de dire que l'équipe de Mens y était en force.

13 Ibid., p. 293.

14 Ramsay Cook, "Le chanoine Lionel Groulx : un "agent double" ?", Mens : revue d'histoire intellectuelle de l'Amérique française, vol. IV, $\mathrm{n}^{\circ} 2$ (printemps 2004), p. 318.

15 Michel Bock, [Compte rendu de Deux Chanoines], Histoire sociale = Social History, vol. 37, n 74 (novembre 2004), p. 271. 


\section{Dix ans d'auteurs et d'articles}

Il est temps de nous pencher sur ce qui a constitué le cœur de la revue pendant ces dix années : les auteurs et leurs articles. La liste des auteurs nous a permis d'en recenser 62, soit une moyenne de trois par numéro (nous analysons ici les vingt premiers numéros). La première chose qui frappe, c'est que pratiquement aucun auteur ne revient deux fois : il y a donc grande variété à ce chapitre. On y trouve - c'est la distinction la plus facile à faire -43 hommes et 18 femmes : la prépondérance du masculin demeure. La seule qui a publié deux articles est Louise Vigneault, une historienne de l'art, qui a écrit sur Zacharie Vincent (printemps 2006) et Paul-Émile Borduas (automne 2009).

La très grande majorité des auteurs vient du monde universitaire. Nous les avons répartis entre professeurs, doctorants et mémorants. L'équilibre est assez impressionnant : 23, 19 et 17 respectivement. Un mouvement se dessine au fil des ans : de 2000 à 2002, le plus grand nombre se trouve du côté des maîtrises (8 sur 17), de 2003 à 2005, du côté des doctorats (10 sur 17) et de 2005 à 2007, du côté des professeurs ( 9 sur 13). Le même mouvement se dessine, à plus petite échelle, de 2008 à 2010 : on passe de trois mémorants et deux doctorants (2008) à six professeurs (2009-2010).

La discipline historique tient le haut du pavé : la moitié des auteurs (32) sont rattachés à un département d'histoire ou sont manifestement historiens. Suivent les études littéraires, qui regroupent 10 auteurs. Plusieurs autres disciplines se partagent le reste du gâteau : sociologie, science politique, histoire de l'art, sciences religieuses. Tous les auteurs, sauf un, peuvent être rattachés au Québec ou au Canada, l'exception étant Olivier Dard, de l'Université Paul Verlaine de Metz, qui a publié, à l'automne 2007, un article substantiel (60 pages) et extrêmement fouillé sur le maurrassisme et ses influences ou transferts culturels et politiques vers le Canada français.

La présence du Canada anglais ou du Canada français hors Québec est relativement forte : on peut y rattacher 22 auteurs, soit 
le tiers du corpus, dont le plus grand nombre se trouve à l'Université d'Ottawa (Département d'histoire ou Centre de recherche en civilisation canadienne-française), soit une dizaine, et les autres à l'Université Laurentienne ou à York, pour les francophones, et surtout à l'Université de la Colombie-Britannique ou à Queen's, pour les universités anglaises, l'influence de Michel Ducharme me paraissant ici évidente (je n'ai pas compté chez les anglophones les francophones rattachés à McGill ou à Concordia). On prendra d'ailleurs mes données avec quelques pincettes, car on se rend bien compte qu'il n'est pas facile de catégoriser chacun des auteurs, à moins de les connaître personnellement.

Tournons-nous maintenant vers les articles. On est d'abord frappé - mais c'est sans doute un peu normal en histoire intellectuelle - par le grand nombre d'articles qui traitent d'un auteur ou d'un personnage : il y en a 24 sur 56 , une fois qu'on a exclu les six articles publiés sous la rubrique "Perspectives». L'entre-deux-guerres se taille la part du lion, avec le tiers de ces articles, où les membres du clergé sont les plus nombreux : Lionel Groulx, Joseph-Papin Archambault, Louis Lachance, François Hertel et Alfred Baudrillart voisinent avec Esdras Minville, Harry Bernard et Hector de SaintDenys Garneau. Pour la période qui précède, on peut citer six articles : Fleury Mesplet et Valentin Jautard, Zacharie Vincent, Alexis de Tocqueville, Edmond de Nevers, Jules Helbronner, M ${ }^{\mathrm{gr}}$ Bruchési et Albert Laurendeau. Et pour la période qui suit, outre Borduas, Mens nous a présenté des articles sur des hommes politiques (Pierre Elliott Trudeau, Claude Ryan, Roy McMurtry, auxquels on peut ajouter Raoul Roy) et des essayistes (Jacques Grand'Maison, Gilles Leclerc, Pierre Vadeboncœur, et un débat entre Jean Larose et Jacques Pelletier). Des figures à peu près toutes assez bien connues, d'où l'intérêt du lecteur à en apprendre davantage à leur sujet.

Nous avons tenté de classer les 32 autres articles géographiquement et chronologiquement. Du point de vue de l'espace, ils traitent tous du Québec ou du Canada français, quoique souvent en relation avec d'autres espaces (France, Europe, Irlande, Allemagne, Belgique, 
Afrique du Sud). Le Canada français et l'Acadie s'y taillent une place respectable : six articles peuvent s'y rattacher. Quatorze des 32 articles se situent exclusivement au Québec. L'insistance sur le Canada français représente un choix de la revue. Deux membres du comité de rédaction, Damien-Claude Bélanger et Michel Bock, s'intéressent directement à cette question et sont tous deux à l'Université d'Ottawa, où se trouve le siège de la revue depuis 2009. En 2005, Mens consacrait un numéro à l'Ontario français et, dans sa présentation, Dominique Foisy-Geoffroy insistait sur la dimension canadienne-française de la revue, dont les mots "de l'Amérique française » dans le sous-titre se faisaient alors l'écho.

Un numéro vient peser assez lourdement sur nos classements : c'est le numéro thématique, beaucoup plus gros que les autres (8 articles, 310 pages, sans inclure les comptes rendus), du printemps 2005 sur l'histoire du livre et de l'imprimé. Un regard sur cet ensemble donne l'impression qu'il aurait mieux valu le publier comme livre : il en a l'unité et la taille. Et il me semble que ces articles font un peu bande à part dans l'ensemble des 56 articles de la revue, du fait qu'ils sont issus des travaux d'une chaire de recherche du Canada. Un genre d'initiative à éviter à l'avenir, à mon avis, pour conserver à la revue sa visée première et son caractère régulier, tant pour la taille que pour le contenu.

Quand on regarde la période couverte par nos 32 articles restants, quatre précèdent le $\mathrm{XIX}^{\mathrm{e}}$ siècle, dont trois proviennent justement de ce numéro sur l'histoire du livre. Le $\mathrm{XIX}^{\mathrm{e}}$ siècle n'en contient guère plus : nous en avons relevé cinq, auxquels on peut en ajouter deux qui couvrent à la fois le $\mathrm{xIX}^{\mathrm{e}}$ et le $\mathrm{XX}^{\mathrm{e}}$ siècle. Le premier $\mathrm{XX}^{\mathrm{e}}$ siècle (1900-1950) arrive avec le gros lot : 13 articles, tandis qu'on en trouve 8 pour la deuxième moitié du siècle. Si on résume cette analyse, il est clair que le Québec du premier $\mathrm{xx}^{\mathrm{e}}$ siècle est le sujet de prédilection de la revue Mens pendant ses dix premières années.

Tenter de classer ces articles par thèmes est un peu plus risqué. Mais qui ne risque rien n'a rien! Naturellement, nous avons d'abord les huit articles analysant certains aspects de l'histoire du livre et de 
l'imprimé, auxquels on peut joindre un mémoire sur les Éditions Mille Roches, du Haut-Richelieu. Le thème du nationalisme, qu'il soit traité à travers des revues (La Revue moderne, Vivre, The BeaverCanada First) ou des mouvements (les Jeunes Laurentiens, le mouvement de la bonne entente), occupe une place de choix. On peut placer sur le même pied le thème de la mémoire et de la commémoration, qui revient sous plusieurs formes. Par ordre chronologique, on peut nommer : l'édification des mémoires collectives au CanadaUni, la mémoire des Canadiens français ayant participé à la guerre des Boers, l'érection de la croix du mont Royal en 1924, la commémoration du bicentenaire de la déportation des Acadiens, la mémoire de la francité dans Les filles de Caleb et l'usage du Règlement 17 dans la bataille de l'Hôpital Montfort. Cinq articles tentent de retracer l'influence d'idéologies européennes au Québec : les révolutions européennes de 1848 , le radicalisme français au début $\mathrm{du} \mathrm{xx}^{\mathrm{e}}$ siècle, le maurrassisme, Louis-Ferdinand Céline et Pierre Drieu de La Rochelle, le régime hitlérien. Il reste alors deux articles sur le féminisme, une analyse de trois revues catholiques au moment du concile Vatican II et trois articles de type historiographique. Voilà le portrait d'ensemble.

\section{Quelques autres rubriques}

Que les articles se limitent à l'Amérique française, cela correspond bien au nom de la revue et ne dépend guère du comité de direction : on prend les articles qu'on reçoit. Mais dans les comptes rendus, on peut orienter davantage le cours des choses, et choisir des ouvrages qui ouvrent sur des horizons divers. Nous avons calculé que, pendant ces dix ans, Mens a rendu compte de 164 ouvrages, soit en comptes rendus, soit en notes critiques (appelées dans un premier temps notes de lecture). Examinant les lieux des maisons d'édition de ces ouvrages, nous avons abouti aux résultats suivants. Plus des deux tiers des ouvrages analysés, soit 113 sur 164, ont été publiés au Québec. Vient ensuite le Canada (anglais) hors Québec, avec 30 titres, la France en compte 8, les coéditions avec la Suisse ou la France 6. Les sept autres 
se partagent entre l'Ontario français (2), le Québec anglais (2), la Belgique, les États-Unis et un ouvrage publié à la fois en Angleterre et aux États-Unis. Il y a donc une certaine ouverture.

Mens a aussi publié huit bibliographies, de dimensions et de caractères assez différents. Dans les premiers numéros, on percevait une ambition, celle de présenter l'histoire intellectuelle dans différents univers : le Canada et le Québec (2001), les États-Unis (2002), la France (2003), entre lesquels s'était glissée une bibliographie sur le féminisme au Canada et au Québec (2001). Il va sans dire que le numéro thématique sur l'histoire du livre eut droit à sa bibliographie. En 2005, la revue annonçait qu'elle prenait la suite pour la mise à jour des bibliographies d'Yvan Lamonde sur l'histoire des idées et de la culture au Québec depuis 1760. Le troisième de ces suppléments, préparé par Yvan Lamonde et Éric Leroux, constitua une section reliée à part du numéro de l'automne 2005, de 96 pages, aussi disponible sur Internet. Une bibliographie sélective sur les droites européennes fut publiée en 2006. Enfin, Yvan Lamonde, qui est décidément intarissable en matière de bibliographie, publia avec son étudiante Cécile Facal une bibliographie commentée sur Jacques et Raïssa Maritain au Québec et au Canada français, qui constitue presque un article bibliographique, avec ses 118 pages. Avec le développement rapide des moyens de repérage par voie électronique, on peut se demander quel est l'avenir des bibliographies imprimées. (En tout cas, celles de Mens sont toutes disponibles sur Internet, puisque tous les numéros de la revue s'y trouvent, à part ceux des deux dernières années).

\section{Évolution récente et direction envisagée}

Depuis 2007, la revue a vécu un certain nombre de transformations, qui ont culminé dans la nouvelle toilette et le nouveau sous-titre pour ouvrir la dixième année, à l'automne 2009. D'abord, on annonçait dans le numéro d'automne 2006 l'arrivée de deux nouveaux collaborateurs dans l'équipe de direction, ce qui élargissait beaucoup 
les horizons de la revue. Harold Bérubé préparait alors son doctorat en études urbaines, étudiant les banlieues anglophones de l'île de Montréal, tandis que Martine-Emmanuelle Lapointe venait de soutenir une thèse en critique littéraire, publiée en 2008. Cette même année, Dominique Foisy-Geoffroy devient directeur de la revue, tout en demeurant secrétaire-trésorier. L'équipe continue néanmoins de fonctionner sur le même mode collégial.

C'est avec le numéro d'automne 2009, marquant le début de sa dixième année, que la revue se donne " un nouveau souffle » : « nouvelle toilette, nouveau site Web, réorganisation administrative et même nouveau nom ", " tout en demeurant fidèle à l'esprit qui l'anime depuis sa fondation en $2000^{16}$ ". À mon avis, l'équipe de Mens n'a fait qu'une erreur dans son parcours, et ce péché originel fut le nom de baptême donné à la revue : un mot latin, que personne dans la jeune génération ne peut comprendre, ne connaissant pas le latin. Ce mot mens signifie esprit, comme l'expliquait bien le premier numéro, se réclamant de Cicéron : "Princeps animi pars mens nominatur» (comprenne qui pourra!). Pour le commun des mortels qui s'intéresse à des revues, mens fait plutôt penser à l'abréviation de mensuel. Mais enfin, la continuité a son prix, et pour plus de clarté, le périodique fait sa publicité sous le nom de Revue $M E N S$, qui est également son nom de domaine sur son site Web : www.revuemens. ca. La continuité est ce qu'il y a de plus précieux pour une revue, et le fait que Mens ait conservé son nom, son format, sa périodicité, son noyau de direction et, surtout, son esprit - c'est le cas de le dire ! - est tout à son honneur.

Le changement de nom s'est manifesté dans le sous-titre, qui se lit désormais Revue d'histoire intellectuelle et culturelle. Le champ de l'Amérique française reste présent et l'ajout de "culturelle » fait partie de l'élargissement des perspectives, même si la revue se situait dans le domaine de l'histoire culturelle dès son premier numéro. Plus

16 Léquipe de Mens, "Présentation ", Mens: revue d'histoire intellectuelle et culturelle, vol. X, n 1 (automne 2009), p. 5. 
d'auteurs se sentiront plus à l'aise d'envoyer des articles à une revue qui se réclame de l'histoire culturelle, et maintenant que la limitation géographique a disparu du sous-titre, on peut s'attendre à plus d'articles touchant d'autres aires que le Québec ou le Canada français.

Autre changement important : la revue, qui était passée de Montréal à Québec en 2002, a maintenant pignon sur rue à l'Université d'Ottawa, en lien avec le Centre de recherche en civilisation canadienne-française (CRCCF) et la Chaire de recherche sur l'histoire de la francophonie canadienne, dont Michel Bock est le titulaire. Ce dernier et Damien-Claude Bélanger étant professeurs au Département d'histoire de cette université, et Dominique FoisyGeoffroy œuvrant désormais également à Ottawa, à Bibliothèque et Archives Canada, ce changement est allé de soi et a été facilité par la collaboration d'Yves Frenette, alors directeur du CRCCF. Il y là un alignement des planètes qui promet.

L'autre grande nouveauté est la refonte du site Web de la revue. Au moment d'écrire ces lignes, la consultation du site de la revue ne manque pas de piquant. Qu'on me permette un moment de récréation : en écrivant " revue mens " dans le moteur de recherche le plus connu, on tombe sur une série de sites à couleur toute spéciale. Le plus voyant s'intitule "Images correspondant à revue mens ". On voit là des photos des revues Men's Health, "le magazine du bien-être au masculin ", et Men's Vogue, puis d'affriolantes jeunes filles et, enfin, la couverture de notre docte revue, dans ses deux moutures. Assurément, le marketing de la revue est assuré ! Qu'on se rassure : Google renvoie d'abord à quatre adresses qui désignent bien notre revue $M E N S$.

Dans son état actuel, ce site est précieux. Il donne d'abord une description de la mission de la revue, tirée du premier numéro, à laquelle s'ajoutent les éléments pertinents de la présentation du numéro de l'automne 2009. Les bibliographies déjà publiées par la revue y sont disponibles, ce qui rend leur consultation beaucoup plus pratique et utile. Une notice détaillée sur chacun des membres du comité de direction est tenue bien à jour : c'est là que j'ai vu que Nova Doyon fait désormais partie du comité. Son agrégation confirme 
l'ouverture à la fois vers la littérature - elle est membre du Centre de recherche interuniversitaire sur la littérature et la culture québécoises (CRILCQ) et spécialiste d'histoire littéraire comparée - et vers les femmes, le comité étant désormais plus équilibré à cet égard. En juillet 2010, on apprend que Joel Belliveau, historien de l'Université Laurentienne (Sudbury) et spécialiste de l'histoire intellectuelle acadienne, en fait également partie. Dans la rubrique "Quoi de neuf ", on trouve des annonces de colloques ou des appels de textes, comme celui annonçant l'apparition d'une nouvelle revue électronique en avril 2010, intitulée Histoire engagée, un site Web qui se situe "dans le sillage de l'initiative Internet britannique History \& Policy". En somme, le nouveau site de Mens est un site vivant qui permet de voir la vie de la revue et de son champ entre la parution des différents numéros.

\section{En conclusion...}

Faut-il conclure, à propos d'une histoire en marche? Certes non. Mais après cette revue à peu près complète des différents aspects de Mens, l'auteur de ces lignes se rend bien compte de deux lacunes. Il porte l'entière responsabilité de la première : l'article ne s'est guère attaqué au contenu même de la revue, à la teneur des articles, aux thèses qui y sont développées. Tâche impossible à réaliser ? Sûrement pas, mais je le disais au début, il faudrait sans doute pouvoir compter sur un spécialiste pour le faire.

L'autre lacune de cet article est qu'il n'a pas encore abordé ce qui me parait le sel même de cette revue : son esprit. Je vais essayer d'en dire un mot, me rendant bien compte, malgré tout, que je suis quelqu'un de l'extérieur. Ceux qui vivent l'aventure de l'intérieur pourraient sans doute en parler plus et mieux, mais on admettra qu'à ce stade-ci, ils sont peut-être moins bien placés pour le faire.

Se rend-on compte, pourtant, de ce que c'est que d'entreprendre, à cinq ou six étudiants de maîtrise, de publier une revue, régulièrement, deux fois par année, avec tout ce que cela peut représenter de démarches, 
d'efforts, de rencontres, d'échanges, tant du point de vue matériel qu'intellectuel ? Évidemment, il y faut une passion et un esprit d'équipe hors du commun. Je crois que c'est ce que l'équipe de Mens a le mieux réussi à réaliser. Numéro après numéro, analyser les articles reçus, solliciter des auteurs pour les comptes rendus, prendre contact avec tel ou tel pour un numéro thématique, décider des rubriques à maintenir ou à supprimer (par exemple, au début, il y avait une rubrique de publication d'inédits, qu'on a vite abandonnée). Et je ne parle pas des tâches d'édition : relire les manuscrits, suggérer des révisions, corriger la langue, surveiller les coquilles, voir à la mise en page, relire les épreuves. Mais où trouver le temps de faire tout cela, quand on rédige sa thèse de doctorat? Le temps vient du feu sacré. Indéniablement, à lire les présentations de chaque numéro, on est frappé justement par le feu sacré et le dynamisme - le dynamisme de la jeunesse, sans doute - qui animent les membres de cette équipe. Et c'est ce qui donne plaisir à lire chaque numéro : on peut voir comment, encore une fois, ils ont réussi l'exploit.

Et puis, il y a une autre dimension. On aurait pu craindre que, dans un champ si miné, l'unité de l'équipe n'explose sur des grenades placées sur son chemin, ou encore que les membres ne s'enferment dans un sillon trop étroit, idéologiquement marqué. On doit reconnaître que tel n'a jamais été le cas et que toutes les écoles, toutes les avenues, sont ouvertes et bienvenues à Mens. Ce n'est pas son moindre mérite.

Devant toutes les exigences d'une telle publication, la nouvelle organisation devrait faciliter la tâche. D'abord, l'équipe est plus nombreuse : on peut se partager les travaux. Et puis, on trouve au CRCCF des personnes d'expérience, habituées à publier des revues savantes. L'union fait la force, c'est le cas de le dire.

Il reste qu'à la fin d'un tel bilan, on aimerait aussi connaître un peu la vie de la revue, vue de l'intérieur. On me dit, par exemple, que les finances de la revue sont " glorieuses " (les guillemets sont de moi, pas des responsables !). Elles sont sans doute parmi les rares 
revues à être dans ce cas par les temps qui courent, mais comment ne pas s'en réjouir ? Et tant d'autres aspects pourraient être abordés, comme les projets ou les rêves...

\section{Dernière heure : un article de Martin Petitclerc sur l'histoire des idées}

J'ai remis la première version de cet article à la revue le $1^{\text {er }}$ juin 2010. Or voici que la semaine suivante paraît dans la Revue d'histoire de l'Amérique française (été 2009), qui prend ainsi la pole position dans les débats en histoire, un article fouillé et stimulant de Martin Petitclerc critiquant le retour de "Notre maître le passé » et réfléchissant sur la crise de l'histoire sociale et l'émergence d'une nouvelle histoire des idées. Son solide bilan historiographique se déroule en quatre temps. D'abord, le temps des conquêtes, qui voit le triomphe de l'histoire sociale, dans les années 1970 et 1980. Il y distingue deux variantes, l'une dominante, la tendance moderniste, l'autre minoritaire (c'est la sienne, celle de l'école de Jean-Marie Fecteau), la tendance critique, qu'il prend bien soin de distinguer du marxisme. Vient ensuite le temps des doutes, des remises en question, la principale étant celle de Ronald Rudin, dans les années 1990. C'est à la fin de cette section qu’à mon avis, il dérape quelque peu, en opinant que le renouveau historiographique qui se manifestait alors « se définissait contre l'histoire sociale ${ }^{17} \%$. J'y reviendrai.

C'est alors le temps des rejets, dont la figure principale est Pierre Trépanier, qui veut valoriser la tradition : "Trépanier, écrit-il avec raison, fut l'un des principaux promoteurs du renouveau historiographique concernant l'histoire des idées et des intellectuels ${ }^{18}$. " Et de mentionner les thèses « très importantes » de Xavier Gélinas, d’Éric

\footnotetext{
17 Martin Petitclerc, « Notre maitre le passé ? : le projet critique de l'histoire sociale et l'émergence d'une nouvelle sensibilité historiographique ", Revue d'histoire de l'Amérique française, vol. 63, nº 1 (été 2009), p. 97.

${ }^{18}$ Ibid., p. 101.
} 
Bédard, de Michel Bock et de Damien-Claude Bélanger, de même que l'apparition de la revue Mens (une coquille tronque alors le soustitre de la revue, oubliant le mot histoire). Il s'en prend surtout au collectif dirigé par Stéphane Kelly, Les idées mènent le Québec, qui déguise ses tendances de droite sous le nom de "nouvelle sensibilité ", et particulièrement à l'article de Bédard et Gélinas, "Critique d'un néo-nationalisme en histoire du Québec ", qui n'y allait pas de main morte, en effet, pour fustiger « les penseurs de la Révolution tranquille et d'après [d'avoir] bradé la tradition d'ici ${ }^{19}$ " au nom de la modernité.

Dans un dernier temps, Petitclerc retrace le contexte du temps de la fidélité, celui qui veut remettre à l'honneur la tradition nationale. On pense aux sociologues Jacques Beauchemin ou Joseph Yvon Thériault. Le risque de ce genre d'histoire qui s'interdit " de porter un jugement sur les idées et les valeurs $[\mathrm{du}]$ passé " est " de se faire essentiellement serviteur de la mémoire ${ }^{20}$ ". Quant à lui, Martin Petitclerc appelle " au renouvellement d'une perspective critique » et veut " réactualiser le projet critique de l'histoire sociale ${ }^{21}$ ".

Beau programme, auquel nous voulons bien applaudir des deux mains. Mais on me permettra de réagir brièvement à certaines des affirmations de l'auteur. D'abord, cette idée que l'histoire sociale est attaquée de toutes parts. Il est vrai qu'elle régnait sans partage dans les années 1970 et 1980 et que depuis le milieu des années 1980, c'est l'histoire culturelle qui a pris le relais de cette domination. Cependant, cette histoire culturelle ne veut pas mettre de côté l'histoire sociale : au contraire, elle tente de s'y ancrer, comme le montre bien le titre du grand projet du principal historien de cette tendance, Yvan Lamonde, Histoire sociale des idées au Québec (quoique le deuxième tome de cet ouvrage (Fides, 2004) ait perdu le peu de dimension

19 Éric Bédard et Xavier Gélinas, "Critique d'un néo-nationalisme en histoire du Québec", dans Kelly (dir.), Les idées mènent le Québec, p. 80 (propos attribués à Fernand Dumont).

20 Petitclerc, " Notre maître le passé? ", p. 113.

21 Ibid. 
sociale qu'on trouvait dans le premier (Fides, 2000). En somme, cette histoire des idées est beaucoup plus politique que sociale).

Il n'est pas surprenant que dans cette foulée de l'essor de l'histoire culturelle, l'histoire intellectuelle - plus large, en passant, que l'histoire des idées - ait trouvé sa place toute naturelle. Et la revue Mens est la principale manifestation de la vitalité de cette histoire intellectuelle, depuis l'an 2000. Il est vrai également que cette histoire est apparue sous l'influence de Pierre Trépanier, adepte d'une idéologie traditionaliste de droite, pour ne pas dire d'extrême droite (Maurras, Rumilly) : les trois principaux animateurs de la revue, entre 2000 et 2010, Damien-Claude Bélanger, Dominique Foisy-Geoffroy et Yves Bégin, ont tous complété leur maîtrise avec Trépanier en 2000-2001, au moment même où ils lançaient la revue.

Mais ce qui est admirable, à mon sens, c'est que, dès le début, l'équipe de Mens, sans jamais renier ses racines, a élargi son spectre pour toucher toutes les sphères de l'histoire intellectuelle, du mouvement ouvrier aux luttes féministes, en passant par les rébellions. Toutes les tendances idéologiques y sont bienvenues et c'est évidemment ce qui fait la force de la revue. L'élargissement du comité de rédaction en 2007, avec l'arrivée d'Harold Bérubé et de MartineEmmanuelle Lapointe, agrandit encore l'horizon et rend les critiques de Mens par Petitclerc complètement dépassées. C'était peut-être vrai dans le manifeste de 2000 comme dans le colloque de McGill de 2000 qui a abouti à l'ouvrage Les idées mènent le Québec : ce ne l'est plus guère maintenant, et personne, aujourd'hui, en lisant la revue Mens, ne peut avoir l'impression d'une " histoire des idées qui suivrait son cours " malgré » le pouvoir, l'exploitation et les inégalités ${ }^{22}$ ".

Le nouveau sous-titre de la revue, Revue d'bistoire intellectuelle et culturelle, comme l'ajout de littéraires au comité de rédaction, consacre cet élargissement et cette volonté de voir grand, dans un esprit d'ouverture et de découverte. Mens est ainsi appelée à prendre une place encore plus grande parmi nos revues d'histoire et à être le 
premier porte-parole de l'histoire culturelle. Il est vrai que l'histoire sociale critique n'a pas de flambeau de pareille stature, ni même de véritable lieu d'expression. Malgré des articles de grande qualité, la revue Histoire sociale ne paraît pas représenter ce courant - ni aucun autre d'ailleurs - tant elle ratisse large. Elle triomphait au moment où l'histoire sociale occupait toute la place en histoire ; elle reste ouverte à tous aujourd'hui sans qu'on puisse y déceler un courant quelconque (à moins que je me trompe...). Le courant de l'histoire sociale critique, représenté par l'équipe du Centre d'histoire des régulations sociales, malgré ses innombrables subventions, ses nombreuses thèses de doctorat et ses publications de grande qualité, n'a donc pas de revue ou de lieu de production phare.

L'histoire intellectuelle et culturelle, pour sa part, a trouvé son lieu naturel, la revue Mens, grâce à une équipe dynamique, qui se renouvelle, une équipe ouverte qui adore ce qu'elle fait et le fait bien, modestement, avec les moyens du bord, mais avec une constance et une ferveur qui imposent le respect.

\section{Et on reprend la conclusion}

Au terme de ce bilan, on peut souhaiter que dans dix ans, la maturité venue, l'un ou l'autre, ou peut-être l'ensemble des membres de l'équipe s'attaque à une rétrospective des vingt ans de la revue qui pourra aller au fond des choses. Mens vient de vivre dix ans d'une enfance heureuse. Elle s'avance dans l'adolescence : celle-ci sera-t-elle tumultueuse ? C'est un âge où les prédictions sont aventureuses. Mieux vaut regarder l'équipe de direction. Les dix premières années ont été celles où ses membres ont, pour la plupart, rédigé leur thèse de doctorat. Maintenant, la plupart occupent des postes universitaires, avec leurs multiples obligations, mais aussi des contacts élargis. Ils sont aussi à l'étape où la conciliation famille-travail peut poser des défis redoutables. Souhaitons que le même esprit qui les a animés jusqu'ici, l'esprit de Cicéron sans doute (« La principale partie de 
l'âme s'appelle l'esprit ", mens), les guide encore sur cette voie de la recherche. Ce qui n'empêche pas, cependant, d'analyser le substrat socioéconomique où s'enracinent ces idées.

La première phrase de l'article de Catherine Pomeyrols sur le Québec dans L'histoire des intellectuels aujourd'hui se lit ainsi : "L'histoire des intellectuels n'existe pas en tant que catégorie historiographique au Québec, alors que l'histoire culturelle y est particulièrement développée ${ }^{23}$. " Et elle se référait à l'article historiographique de 1997 d'Yvan Lamonde. C'était à l'automne 2001. Sans doute que l'auteure de Nantes n'avait pas encore vu la revue Mens, apparue l'automne précédent. Elle ajoutait cependant : "L'absence de rubrique spécifique et l'utilisation limitée du terme ne signifient pas cependant l'absence de l'objet, puisque de nombreuses études font apparaitre la figure de l'intellectuel ${ }^{24}$. "Sans doute que depuis dix ans, la revue Mens a beaucoup contribué à faire apparaître la figure de l'intellectuel. Et qu'en 2010, Pomeyrols n'introduirait sans doute plus son bilan par la même phrase. La revue Mens tiendrait une place de choix dans l'évolution de la discipline. C'est d'ailleurs ce qu'elle laissait déjà entendre dans sa communication, dont voici la finale : "La question de l'intellectuel me semble pouvoir devenir au Québec un objet d'étude à part entière [...]. Elle se situe, en effet, à la jonction de terrains déjà bien défrichés au Québec et devrait permettre dans une perspective d'histoire comparée d'enrichir les questionnements ${ }^{25}$."

On souhaite à Mens, à l'aube de ses dix ans, de poursuivre les défrichements, d'approfondir les questionnements et de continuer à nous fournir, semestre après semestre, cette petite revue dynamique, substantielle, accessible, qui nourrit cette principale partie de l'âme qui s'appelle l'esprit.

\footnotetext{
23 Catherine Pomeyrols, "Comment fait-on l'histoire des intellectuels au Québec?", dans Leymarie et Sirinelli (dir.), L'histoire des intellectuels aujourd'hui, p. 107.

24 Ibid.

${ }^{25}$ Ibid., p. 121-122.
} 